\title{
3D MODELS FOR ALL: LOW-COST ACQUISITION THROUGH MOBILE DEVICES IN COMPARISON WITH IMAGE BASED TECHNIQUES. POTENTIALITIES AND WEAKNESSES IN CULTURAL HERITAGE DOMAIN
}

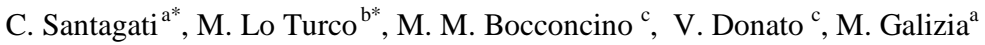

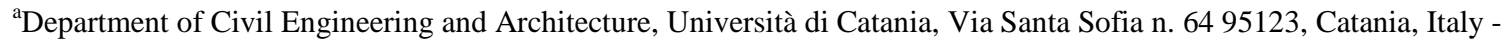 \\ (cettina.santagati, mgalizia@dau.unict.it) \\ ${ }^{\mathrm{b}}$ Department of Architecture and Design, Politecnico di Torino, viale Mattioli n.39 10125, Torino, Italy - \\ massimiliano.loturco@polito.it \\ ${ }^{c}$ Department of Structural, Geotechnical and Building Engineering, Politecnico di Torino, corso Duca degli Abruzzi, 24, 10129, \\ Torino, Italy - (maurizio.bocconcino, vincenzo.donato@polito.it)
}

\section{Commission II}

KEY WORDS: Structure from Motion, Image Based Modelling, Mobile devices, 3D Imaging, Representation Techniques

\begin{abstract}
:
Nowadays, 3D digital imaging proposes effective solutions for preserving the expression of human creativity across the centuries, as well as is a great tool to guarantee global dissemination of knowledge and wide access to these invaluable resources of the past. Nevertheless, in several cases, a massive digitalisation of cultural heritage items (from the archaeological site up to the monument and museum collections) could be unworkable due to the still high costs in terms of equipment and human resources: 3D acquisition technologies and the need of skilled team within cultural institutions. Therefore, it is necessary to explore new possibilities offered by growing technologies: the lower costs of these technologies as well as their attractive visual quality constitute a challenge for researchers. Besides these possibilities, it is also important to consider how information is spread through graphic representation of knowledge. The focus of this study is to explore the potentialities and weaknesses of a newly released low cost device in the cultural heritage domain, trying to understand its effective usability in museum collections. The aim of the research is to test their usability, critically analysing the final outcomes of this entry level technology in relation to the other better assessed low cost technologies for 3D scanning, such as Structure from Motion (SfM) techniques (also produced by the same device) combined with dataset generated by a professional digital camera. The final outcomes were compared in terms of quality definition, time processing and file size. The specimens of the collections of the Civic Museum Castello Ursino in Catania have been chosen as the site of experimentation.
\end{abstract}

\section{INTRODUCTION}

The Anthropocene age is characterised by natural and anthropic hazards (climatic changes, catastrophic events, terrorist attacks, oblivion, destruction) that put in danger the transmission of cultural heritage inheritance of future generations.

3D digital imaging can constitute an effective solution for preserving the expression of human creativity across the centuries, as well as is a great tool to guarantee global dissemination of knowledge and wide access to these invaluable resources of the past.

Nevertheless, in several cases, a massive digitalisation of cultural heritage items-from the archaeological site (Kersten et al., 2012a) up to the monument and museum collections-could be unworkable due to the still high costs in terms of equipment and human resources: 3D acquisition technologies and the need of skilled team within cultural institutions (Remondino, 2011).

Therefore, it is necessary to explore new possibilities offered by growing technologies developed and made available in the market each day.

In this regard, the last developments in the field of Computer Vision and 3D scanning have led to the spread of low cost and fast handheld devices (i.e. Structure Sensor, mobile apps) able to create 3D textured models of real life objects (Lachat et al., 2017).

The easiness of use and availability in the current market will bring in the next years a very massive use of these devices in different fields of application. Nowadays, even if the development of the technology is at a low Technology Readiness Level (TRL); in some cases, the use and experimentation of such technologies could help developers to find and open new fields of research and applications.

The lower costs of these technologies as well as their attractive visual quality constitute a challenge for researchers: they start to experiment with these devices, pushing to the limits their use, trying to understand novel yet unexplored fields of application. The focus of this study is, hence, to explore the potentialities and weaknesses of a newly released low cost device (i.e. Sony Xperia XZ1 and the new app $3 D$ Creator) in the cultural heritage domain, trying to understand its effective usability in museum collections.

As an entry level of technology, it can be within the reach of everyone, so it could constitute a good way for quickly 3D digitising museum collection items and diffusing them on the web for valorisation and dissemination purposes enabling their access to huge public, as well as it could be a resource in projects that foresees the engagement of citizens and communities who could play an active role in the co-creation of 3D contents experimenting a new kind of cultural visit (Inzerillo, Santagati, 2016). Furthermore, we could envision the benefits for the so-called creative industries sector favouring innovative approaches in terms of new products and new business models in the fields of entertainment, digital collections, virtual museums, etc.

The study will verify the $3 \mathrm{D}$ acquisition pipeline in the presence of a set of museum items made by different materials and dimensions in order to identify a possible field of application and set up an optimal workflow. Furthermore, it will carry out a comparison with the well-known and used low cost image based 
modelling pipeline to explore the respective strengths and limits. The remainder of the paper is organised as follows: Section 2 will be dedicated to the analysis of the state-of-the-art. Section 3 describes the methodology and workflow. Section 4 will introduce the case study according to the identified methodology; the results of the comparison will be analysed and discussed in Section 5, then concluding remarks and future activities (Section 6) will complete the work.

\section{CURRENT DEVELOPMENTS AND NEW TECHNOLOGIES}

Nowadays, there are several main classes of 3D non-contact active scanners incorporated in handheld or easily movable device techniques; these are generally classified in: time-offlight, triangulation laser scanner, structured light scanner and modulated light scanner (Apollonio et al., 2017). Although this classification provides a high range of possibility, in literature there are few samples of low cost application, generally due to the limited achievable precision. Usually low cost techniques are developed for entertainment and gaming purposes, requiring lean models and fast rendering elaborations. Only few recent studies (Allegra et al., 2017) have shown some accuracy evaluation between professional tools and low cost techniques. Regarding more professional application, there are several solutions of handheld 3D scanners used to acquire 3D geometry that use triangular light (for instance, Artec's Eva 3D scanner, Portable 3DZCorporation, NextEngine 3D Scanner Ultra HD, Creaform Handyscan 3D, Fuel3D Scanify 3D Scanner, IIIDScan PrimeSense 3D scanner). Then, the workflow is completed by proprietary software, frequently provided by the scanner's manufacturer. There are also some low cost versions of scanners (i.e. Cubify Sense 3D Scanner, MakerBot Digitizer 3D Scanner, EinScan-Pro+) according to the scale of the object that needs to be acquired. Similar solution could be found in structured light techniques (i.e. HP 3D sensor, Rangevision 3D scanner and DAVIS SLS-3 Structured Light 3D Scanner) providing a high-level precision and geometrical accuracy of the acquired objects with lower cost, if compared to the handheld scanner.

Looking at other products, with a specific regard to the latest devices presented at IFA Berlin (Internationale Funkausstellung Berlin is one of the oldest industrial exhibitions in Germany, held in early September, 2017), we can find commercial products that natively implement photomodelling technologies directly integrated into commonly used communication tools usually applied to different purposes (tablets, smartphones). Within this scenario, some tools for automatic reconstruction of the 3D model are now available: some of these make use of photogrammetric approach directly implemented through a dedicated app for tablets and smartphones (1); some others mount additional sensors that can be connected on external devices (2).

The first approach (1) does not require extra hardware: it is based on 3D scanning apps that make use of specific computational geometrical algorithms and can provide optimum solution for an automatic detection of pixel that matches with the same physical point (Trnio, Scann3D, Scene, ReCap 360, Itseez3D, Mobile fusion, 123D catch, 3DF zephyr, Scandy App Pro, only to name a few).

The second approach (2) requires additional sensors (such as Occipital Structure Sensor, Intel Real Sense, EORA 3D) that need to be connected with handheld devices. The technology that gives the ability to understand the distance to objects in the real world is defined as "Depth Perception": the use of an RGB camera, an infrared (IR) projector and IR sensor (Pagliari et al., 2015) are required. Manufacturers' devices can choose from common depth technologies, including Structured Light, Time of Flight, and Stereo (Smisek et al., 2011), in order to implement Depth Perception. Moreover, specific apps have been developed to elaborate these data. Additionally, some low cost devices, originally designed for entertainment and gaming purposes, can be used for digital acquisition: i.e. the Microsoft's Kinect (Smisek et al., 2011), ASUS Xtion and PrimeSense. These platforms need a software package for capturing full colour 3D scans (such as Skanect, FlexScan3D, ReconstructMe and many others). There are several samples on the internet that refer to the use of tablets with Skanect.

Looking at some specific applications and the literature review related to the implementation of low cost techniques in the field of cultural heritage, several authors describe different methods and experiences for the reconstruction of outdoor archaeological sites (Silberman, 2011) and movable archaeological artefacts, in particular referred to the use of SfM approach (Benedetti et al., 2010; Clini et al., 2016, Kersten et al., 2012b; Evgenikou et. al, 2015).

Interesting approaches refer to the use of Kinect applied for the reconstruction of cultural heritage (Smisek et al., 2011; Richards-Rissetto et al., 2012; Bostanci et al., 2015). In not-related fields, sensors are applied for scan body (Tong et al., 2012), or medical purposes, i.e. the implementation of low cost techniques to measure the Human Body Posture (Kurzydlo et al., 2014). Finally, in the field of vehicle industry, especially regarding shape self-recognition, several researches have been implemented for detecting shape low cost sensor (Ward et al., 2016; Vivacqua et al., 2017).

\section{METHODOLOGY}

The aim of the research is to test the usability of $3 D$ Creator app, which is embedded in Sony Xperia XZ1 mobile device (released in Italy, October 2017), in the cultural heritage domain, with particular reference to the creation of virtual museum collections, easily spreadable and shared with an extremely large audience. Indeed the app is mainly designed to develop models and avatars for entertainment applications.

The entertainment nature of this app allows for:

- easy usability of the application;

- fast learning time and confidence with the tool;

- quick acquisition survey procedures;

- low acquisition time (in relation to the size and complexity of the object);

- real-time outcomes and sharing of the model;

- digitally textured model explorable within the application (with a reduced digital size) and common visualisation tools.

These specific features may become strengths when a very large number of objects need to be acquired and the time we spend for acquisition and processing becomes a key element. Additionally, the ability to engage large community of people, not necessarily formed on the more technical aspects, would allow for more workforce (institutions, scholars, professionals, citizenship) and raise public awareness of the need to supply historical data archives through accessible and transparent tools (Li et al., 2016). This work critically analyses the final outcomes of this entry level technology in relation to the other better assessed low cost Image Based technologies for 3D scanning in the field of cultural heritage, such as SfM techniques (Agisoft Photoscan) combined with dataset generated by a professional digital camera, Canon EOS 1200D (Figure 1). Additionally, the embedded camera of Sony Xperia mobile device to create SfM 3D textured models was used and tested to create new specific datasets.

The experimental activity has been carried out starting from objects of small dimensions, then trying to increase the size and understanding the physical limit beyond which deformations 
should be considered unacceptable. The experimentation has been done step by step, by referring to geometries of increasing complexity, starting from simpler examples of solid primitives, up to the extreme complexity of the findings exhibited in important museum collections.

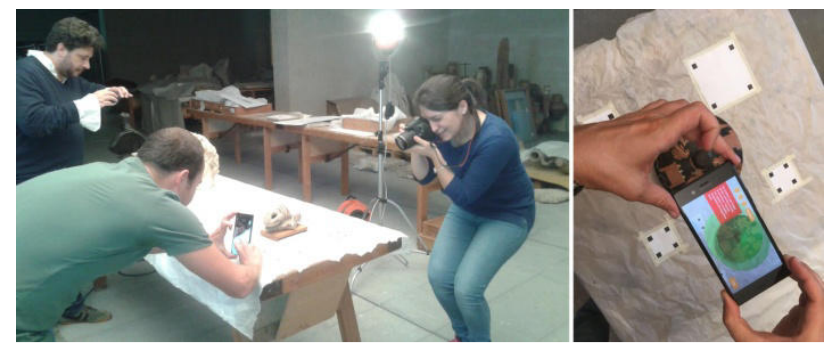

Figure 1. Acquisition phase done with different instruments: Digital Cameras used to prepare image dataset (SFM), on the right; SONY XZ1 smartphone used with the 3D Creator app, on the left.

The experimentation has been carried out according to these steps:

- $\quad$ setting up of the environmental conditions;

- identification of the items;

- 3D acquisition via 3D Creator app;

- dataset acquisition (Xperia ZX1 and Canon EOS 1200 D);

- processing and scaling of SfM data;

- $\quad$ alignment of 3D models;

- Hausdorff distance calculation between the models.

The comparison will be conducted in terms of:

- cost of equipment;

- acquisition and processing times;

- training times (easiness of uses, skills required);

- mesh quality;

- $\quad$ accuracy (Hausdorff distance evaluation);

- accessibility to the acquired data

Thereafter, a first matrix-assessment was produced to point out and highlight advantages and limits of each process (Santagati et al., 2013).

\section{CASE STUdies}

The specimens of the collections of the Civic Museum Castello Ursino in Catania, have been chosen as the site of experimentation. Castello Ursino is the most important museum in Catania for its vastness and variety of the collection, ranging from the Byzantine-style tablets of the 15th century up to the 19th century oil paintings.

4The museum boasts a rich art gallery; additionally, many archaeological findings of the Hellenistic and Roman times, Greek vases, Greek and Roman coins, portals of the thirteenth and fifteenth centuries, engravings, prints, sacred vestments and ornamental artistic objects of different ages. Nevertheless, only a part of the collection is accessible to visitors, while the main part of the collection is stored in the warehouses and not yet catalogued.

This experimentation is part of a wider project started in 2016 and aimed at the 3D documentation, promotion and diffusion of the museum collections on the web and through social media by means of novel and low cost technologies. Another important goal of the project is the testing of innovative co-creation experiences for the visitors, engaging them in a sort of urban game (\#3Ddigitalinvasion) for the creation of 3D models (Figure 2), by means of low cost technologies such as SfM techniques (Chiabrando et al., 2017) related to the collections during the visit and the sharing on the web. The students of University of Catania have been chosen as target groups (Santagati et. al., 2016).

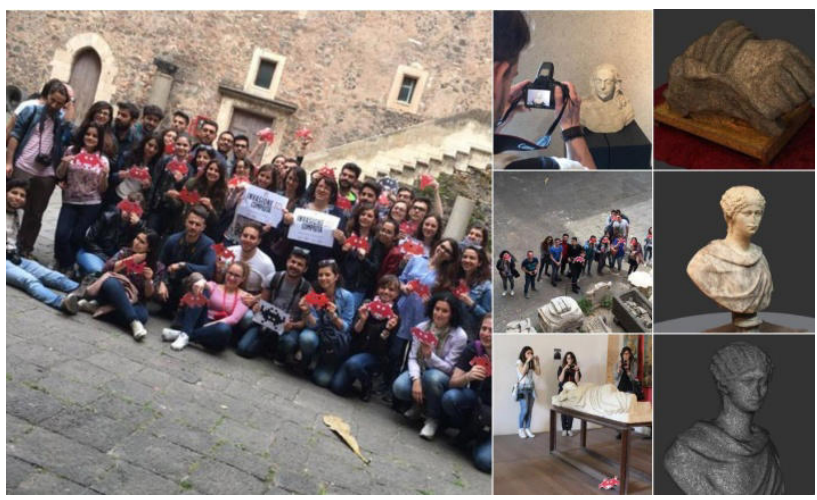

Figure 2. Students from University of Catania engaged in a crowdsourcing activity at Castello Ursino for the creation of 3D models starting from image dataset (SFM).

The work presented here goes in these two directions: the tests were performed both to verify whether it is possible to acquire 3D models stored in the warehouses by using $3 D$ Creator app (trying to understand their quality and usability not only for mere visualisation on the web) and to get 3D models directly during the visit (during which we do not have optimal environmental lighting conditions).

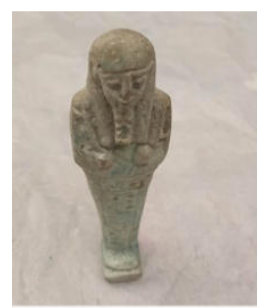

a

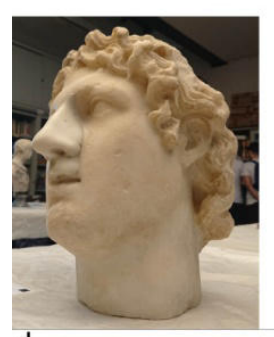

d

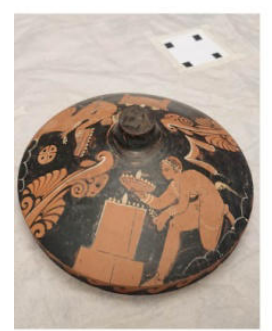

g

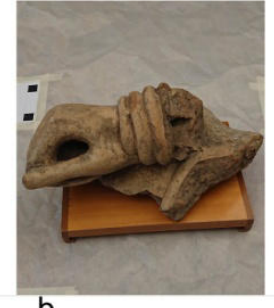

b

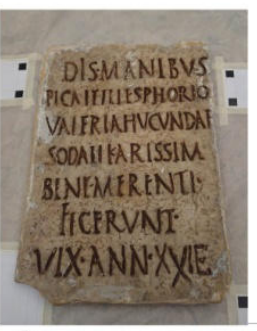

e

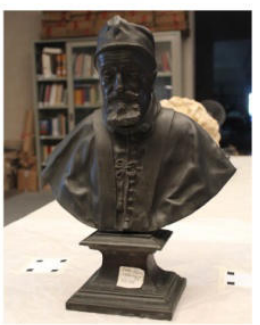

h

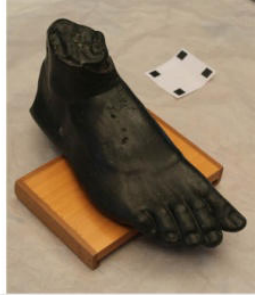

C
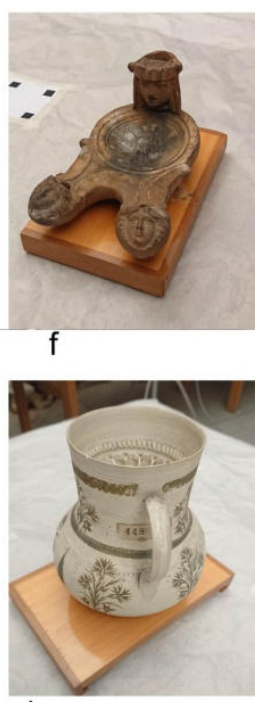

i
Figure 3. a) Egyptian Ushabti (majolica); b) hand (terracotta); c) masculine foot (bronze); d) Head of Alexander the Great (marble); e) Roman Inscription (limestone); f) bicline oil lamp (terracotta); g) Cover of siceliota lekane (pottery); h) Bust of Pope Gregory XV (bronze); i) Samovar for infusion (white terracotta pottery). 
The extreme variety of the collection allowed us for choosing a set of items (Figure 3) stored in the warehouse to enable us to experiment and reason on objects of different dimensions (from 10 to $60 \mathrm{~cm}$ ), materials and surface treatment (marble, limestone, bronze, terracotta, pottery, majolica), shapes and detailed richness. Even the shapes are extremely heterogeneous, from parallelepiped and conical shapes (epigraph, cover), to revolution surface (Samovar) and free form surfaces with holes, recesses, alternation of concavity and convexity, with a detail up to 1-2 millimetres (foot, head, hand, oil lamp).

According to our goal and methodological approach, the variety of materials constituted a great test bench in order to stress the different technologies used and then compared.

\subsection{The 3D Creator app testing procedure}

$3 D$ Creator app has been released with Sony Xperia XZ1 mobile device (the first Italian release is dated October, 2017) and allows to map a 3D image of a very large sample of small objects with a maximum size that should be included in a virtual box of $60 \times 60 \times 60$ centimetres, according to our preliminary tests. The camera includes Autofocus burst, which tracks and captures sequences of moving objects in sharp focus to avoid blurred action shots, and Predictive Capture that attempts to capture unexpected motion.

Regarding the acquisition mode, the application requires some accessibility around the artefacts. The app recognises the object in the scene and begins to detect the coordinates of reference points according to four reading options: spherical (with the commercial diction named "face"), semispherical (named "flat") and food plate sand arbitrary (named "free form") types.

In the first three cases, the application requires that the device has to be moved along a meridional and parallel acquisition path of the sphere identified by the distance from the object to be detected and therefore closely related to the object's dimension. In the third case, the user is free to orbit around the volume. In all three cases, the application provides a series of indications aimed to improve acquisition quality (i.e., not staying too long in a certain position, keeping distances as constant as possible, etc.). These considerations are easily attributable to the need to evenly distribute acquisition errors in order to render more homogeneously the point cloud reliability. After a first scan, the tool allows to increase the number of polygons that define the 3D mesh, especially as a function of critical parts such as small size detail and covered areas.

The interface of the application, in fact, highlights the surface of the digital model with the green colour, and the user assists the construction of the same surface by comparing the forms of the real object traced beyond the screen of the smartphone, with geometric shapes reconstructed by the application algorithm (Figure 4).

Staying on a specific area of the object beyond certain times has a point acquisition limit beyond which bugs and artificially unmanageable forms are introduced; the discretization of the digital surface points is also related to the resolution of the sensor. Conversely, it is not possible to operate on specific acquisition parameters: so the acquisition accuracy settings are not transparent to the users. According to this, it is not possible to distinguish between acquisition phase and three-dimensional reconstruction as the app is able to quickly generate simplified 3D models, thanks to some controlled movement of the smartphone around the object. At the end of the process, the app elaborates the final result in real time. Both the model (*.obj) and the textures can be exported into a zip file that can be open by many applications that work with $3 \mathrm{D}$ elements.
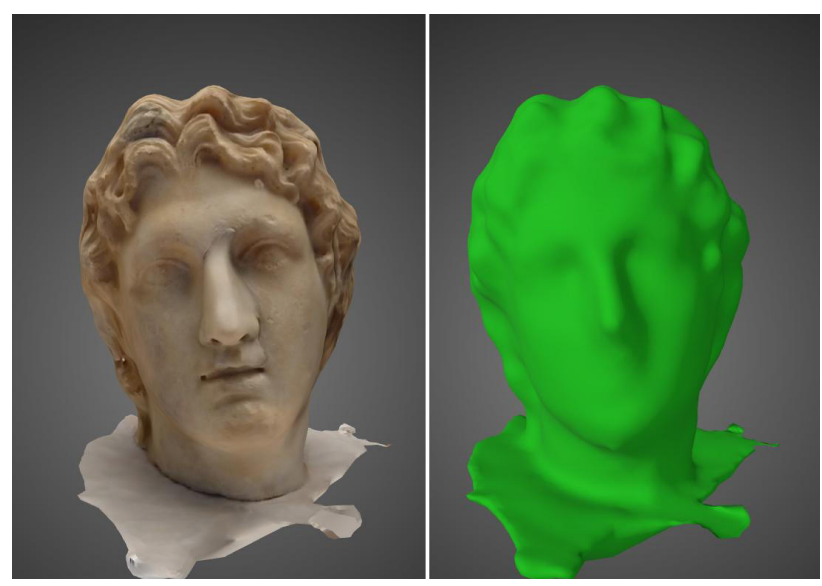

Figure 4. Head of Alexander the Great (marble); on the left, the textured head, on the right the 3D model without the texture. The model is freely available on the web address: https://3dcreator.sonymobile.com/ABrfZFGWYWOyCmLYxU5NQDnQ scl8CCGimJNyWrOnpJZiLzcFXtH18I2_Sx0yBTbxf00jmF9iQp Fd08baTihDTGyGQKIRihU3RNg35_zYckoZq2y7zMxm0PjM eaSGEs6w (last visited: October, 2017).

\subsection{Image Based Reconstruction}

As low cost Image Based Modelling (IBM) software for 3D reconstruction, the well-known Agisoft Photoscan has been used. As known, the IBM techniques are closely dependent on the dataset's quality (network, image resolution, radiometric quality).

We used different kinds of devices-Sony Xperia XN1 mobile and Canon EOS 1200D digital camera-both with an 18 Mpixel resolution.

The setting up of the shooting scene has been designed in order to obtain optimal results. The acquisitions were made in the warehouse rooms. The items have been placed on a table covered with a neutral colour and several markers of known size $(0.05$ and $0.10 \mathrm{~m})$ have been placed in the scene in order to proceed to the next roto-translation and scaling of the digital processing; then particular attention has been given to environmental conditions, the room has been enlightened with artificial spot placed in order to give indirect and uniform light in the room.

Photoscan gives the user the possibility to properly set the parameters of the $3 \mathrm{D}$ reconstruction. The reconstruction takes place in two steps: at first the software performs a 3D alignment between the images and gives back a sparse point cloud, then it is possible to obtain a dense reconstruction where the mesh and the textures are also computed (figures 5,6). The right choice of sparse and dense reconstruction parameters will affect the quality of the 3D model in terms of sharpness of edges and smoothness of surfaces.

In this case, the geometric features of the objects led us to choose a high quality reconstruction for the alignment step and a medium mesh reconstruction in addition with MILD filter. Then we proceeded with the texturing of the models that have been exported in .OBJ format in order to be imported in Meshlab for further processing. 


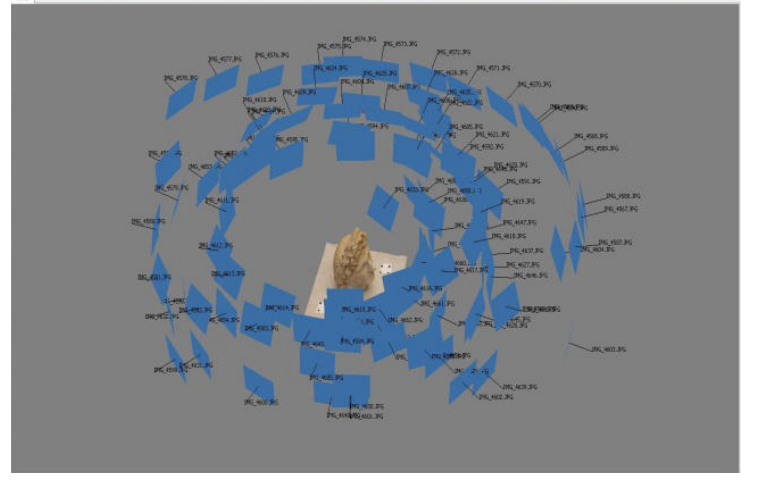

Figure 5. Dataset pose reconstruction of Alexander the Great head

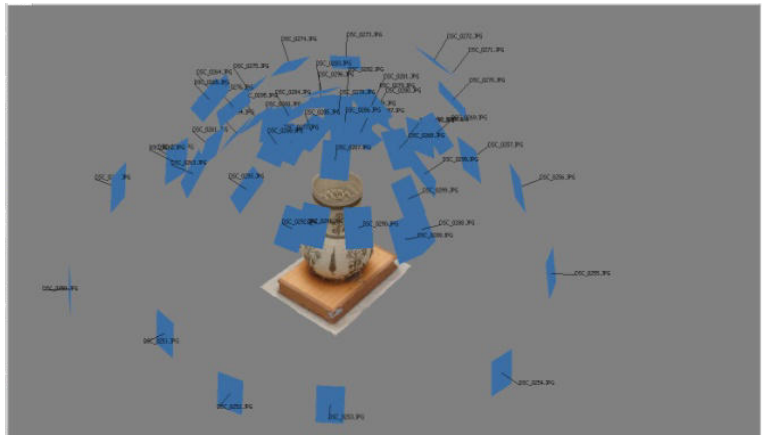

Figure 6. Dataset pose reconstruction of Samovar for infusion

\section{RESULTS}

The 3D textured models obtained by means of 3D Creator app and Photoscan have been compared according to the parameters identified in the methodology. Figures 7, 8, 9, 11 show the differences in terms of appearance (texture), mesh detail (number of faces and shaded model), number of images, processing time and file size.

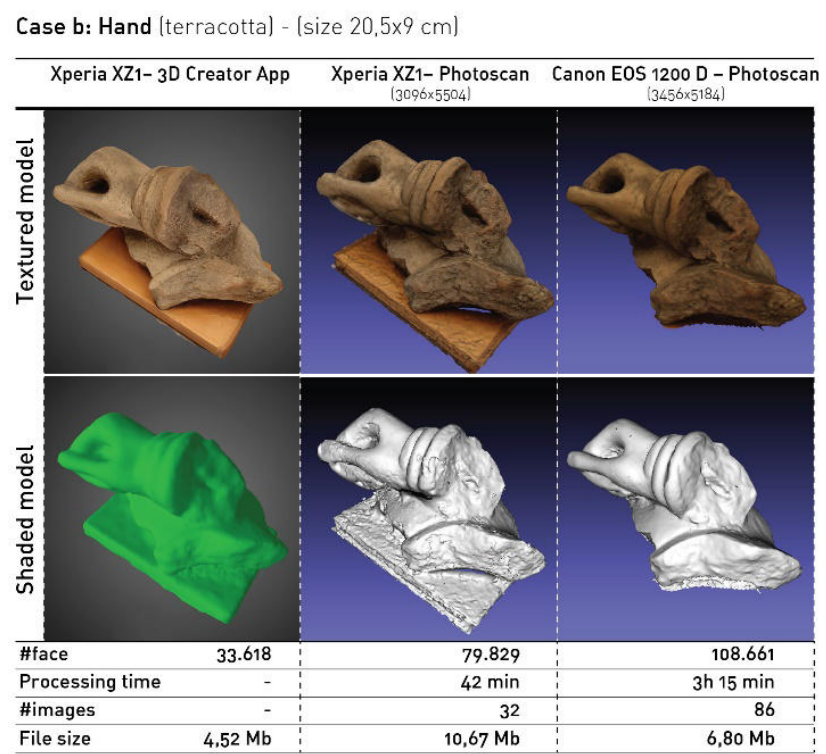

Figure 7. Comparative assessment-matrix on hand

A first consideration that can be done is that even if the level of detail of $3 D$ Creator app is quite low (number of faces), the appearance of the model in textured visualisation is very good and is of the same quality of the other two textured models. This can allow using these kinds of models for visualisation, entertainment and sharing on the web because of the file's lightness.

Regarding another question concerning the smoothing algorithm used by the $3 D$ Creator app, the models are already smoothed, whilst SfM models in some critical points or in presence of reflective materials (bronze, ceramics) are too much noisy.

The low file size and face number is directly connected to the richness of detail as can be observed in the following figures. For instance, in the case of the Roman Epigraph (figure 9), the engraving of the letters is completely shallow.

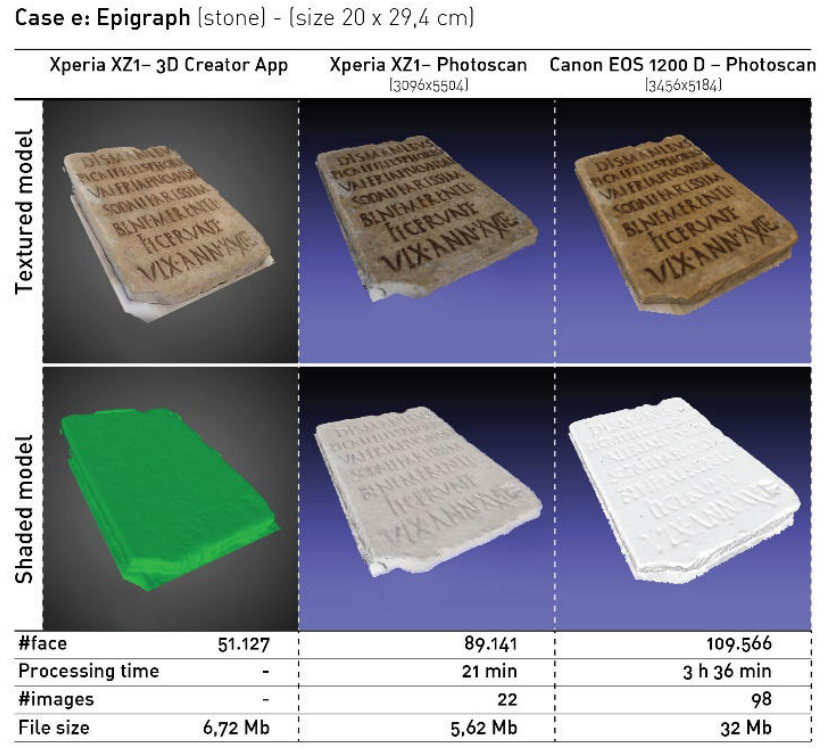

Figure 8. Comparative assessment-matrix on Roman Epigraph

In the following paragraphs, the results of two different specimens-representative of the size, shapes (freeform and conical), materials that can be by found in a museum collectionwill be analysed. We refer to the simple shape of the siceliota Lekane cover in pottery (case g), which is a conical as well as revolution surface and the marbled head of Alexander the Great (case d), which can be considered a freeform surface. In addition to the information that can be obtained from figures 9 and 11, the results of Hausdorff distance calculation will be discussed.

\subsection{Siceliota Lekane cover}

The item belongs to the siceliota production, which is characterised by the use of ceramics with red figures on black background. It is a cover of a Lekane, a flat wide bowl used for serving food or as basin for the water. Its shape could be considered a revolution surface (cylindrical at the base and conical at the top). All the information regarding the acquisition by means of the two technologies is summarised in figure 9 .

Regarding the mesh visual quality, we can observe that the $3 D$ Creator model follows the cover's geometry, even if the knob is not well defined. The SfM model reconstructed using Sony Xperia XZ1 device, is better defined both in terms of geometry and polygons, even if the cover's base presents geometrical reconstruction problems, with a lot of noise. This is probably due to the cylindrical stem's low height. The reflectiveness of the ceramic treatment as well as the black background have caused several problems in the acquisition of the geometry and the texture.

To carry out the comparison, the models have been aligned in Meshlab using Canon EOS 1200 D 3D model as reference. The $3 D$ Creator model has been scaled and aligned by means of 
homologous points exploiting the texture colour. Then, Hausdorff distance between the mesh has been calculated considering an interval range from 0.00 to $0.005 \mathrm{~m}$.

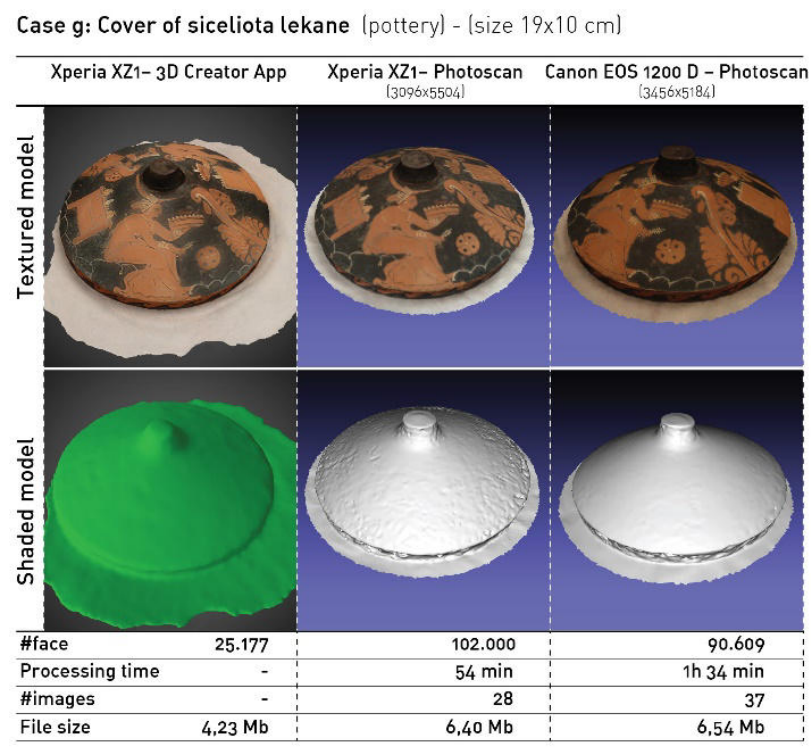

Figure 9. Comparative assessment-matrix on Siceliota Lekane cover

Thereafter, the mesh has been colored by vertex quality: red equals the maximum of the overlap, blue equals the maximum of displacement in the identified range. In figure 10 we can observe the results of two comparisons, on the left 3D Creator model vs. Canon SfM model, on the right Xperia SfM model vs. Canon SfM model. The comparison highlights the geometrical reconstruction errors in the case of $3 D$ Creator model and the differences in terms of noise/smoothing of the surface. In the comparison on the right, we have uniformity in geometrical reconstruction; the main differences are due to the major noise of Xperia SfM dataset in relation to Canon one.

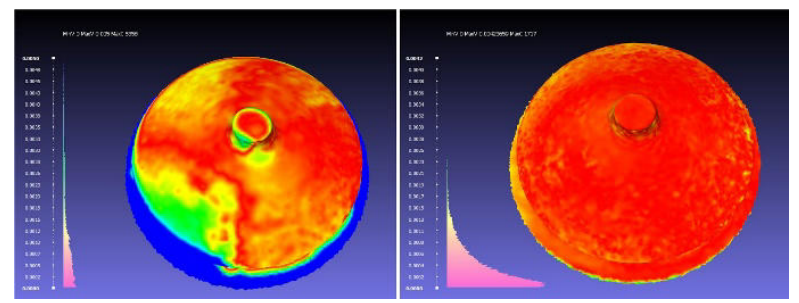

Figure 10. Hausdorff distance calculation between the $3 D$ Creator model and Canon-Photoscan model (on the left), the XperiaPhotoscan model and Canon- Photoscan models (on the right).

\subsection{Alexander the Great head}

The head of Alexander the Great, realised in marble, can be considered a freeform surface with a very smooth face and extremely curly hair. The nose has been restored.

There were no particular problems during the acquisition phase; in the case of $3 D$ Creator, we tested both wizard acquisition procedures for head and freeform mode.

All the information regarding the acquisitions is reported in figure 11. The first consideration that can be done is the difference in richness of details between the $3 D$ Creator app and SfM models, both in the face details, but especially in the curly hairs. However, the visual appearance of textured models is very good. Then, the models were imported in Meshlab for their alignment and
Hausdorff distance calculation. In this case, during the scaling and alignment procedure some difficulties were encountered due to the low detail of the $3 D$ Creator model.

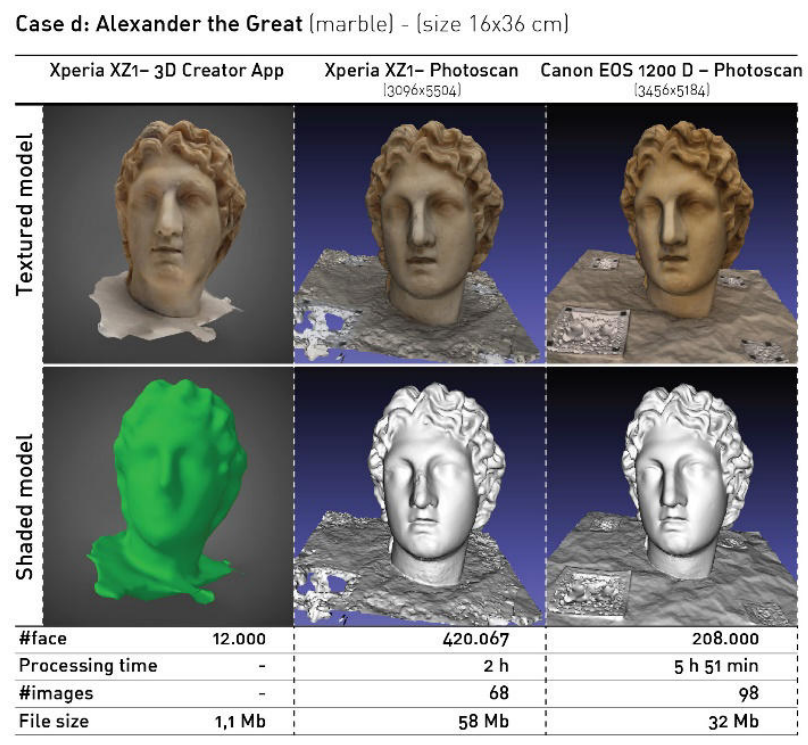

Figure 11. Comparative assessment-matrix on Alexander the Great

The interval range has been set from 0.00 to $0.005 \mathrm{~m}$.

Hence, vertex quality colouration has been applied to the mesh. Figure 12 clearly shows the displacements between the $3 D$ Creator model and SfM model, especially in correspondence of the richness of details.

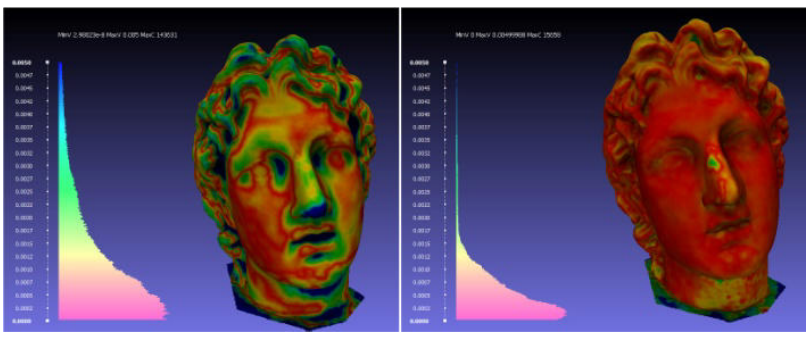

Figure 12. Hausdorff distance calculation between the $3 D$ Creator model and Canon-Photoscan model (on the left), between the Xperia-Photoscan model and Canon- Photoscan models (on the right).

\subsection{D reconstructions: Weaknesses and strengths}

Finally we report emblematic examples of pushing to limits both technologies. The first case is represented by a bronzed masculine foot. In this case, the reflectance of the material leads SFM techniques to failure: the brilliance of surface brings to a very bad dataset, with not homogeneous pixel colours and luminance variability. The result is a very noisy 3D reconstruction (figure 13). On the other hand, the $3 D$ Creator model, even if lacks detail, is sufficiently textured and geometrically defined.

In the second case, we have a Samovar for infusion, realised in white ceramics and decorated with floral figures. The whiteness of the background and its brilliance as well as the thickness of the object leads $3 D$ Creator app to failure. After several attempts, only a portion of the object is acquired. Conversely, we achieved a very good SfM model, with noise problems at the base of the item (figure 14). 

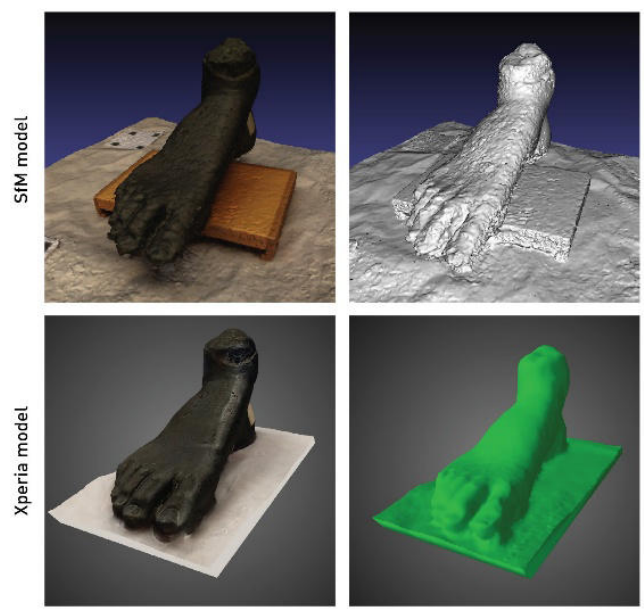

Figure 13. Bronze masculine foot SfM model in comparison to 3 D Creator model
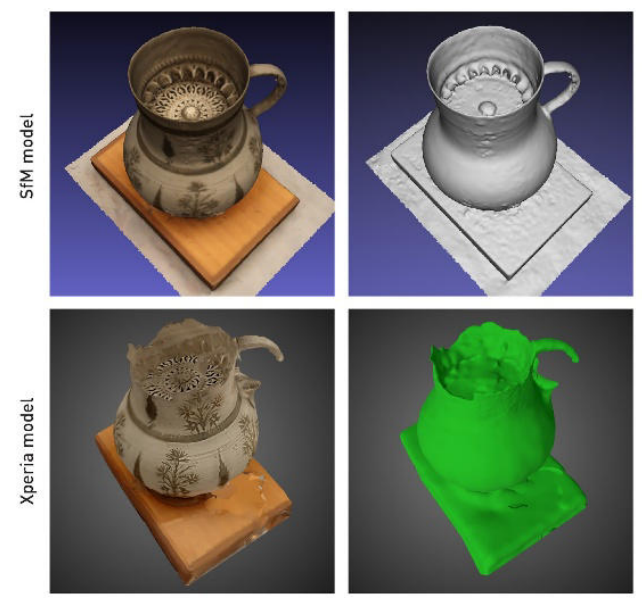

Figure 14. White ceramics Samovar SfM model in comparison to $3 D$ Creator model

\section{CONCLUSION AND FUTURE ACTIVITIES}

In this work we tested a new low cost 3D technology, mainly developed for entertainment purposes, in Cultural Heritage domain.

This first experimentation shows a slight variability in the outcomes, thus we chose to not define backward specific processing thresholds (precision) and acquisition parameters (constraints and good practices). These must be closely linked to the purpose of the survey by specifying ways of detecting the distance and duration of the scan to be related with specific acquisition types:

- environmental factors of the context;

- main dimensions of the modelling object;

- form;

- materials and textures of the surfaces and their physical parameters;

- level of detail (measurement of the minimum parts to be detected).

Dimensions and shapes help to define possible geometric thresholds for use; the materials define colorimetric thresholds; textures and details of the object define detail thresholds. A common problem with this kind of elaboration is the association of a metric reference within the collected photographic images. This reference allows to scale the model and to perform comparisons between scans of different nature.

Regarding the software used, the app creates models not easily controllable in terms of scale factor and proportion between models acquired in deferred times (i.e. it is not possible to reconstruct a homogeneous scale factor from a single model and apply it to models acquired later or earlier).

The 2D markers used during the images acquisition were not collected by the app. A good solution could be to insert in the scene, and close to the object, a 3D geometric shape of known measures, to be considered as reference.

The chance to print the digital copy, by producing a sort of "rapid prototyping" process, has not been tested yet. However, it is possible to point out that in terms of graphic yield related to a use in the publishing/museum domain; the texture greatly contributes to the virtual simulation effect of the object.

In addition to a critical comparison related to the data's nature and accuracy, the time used to produce the models must be measured: on one hand, digital photogrammetry requires short activities in the acquisition phase; on the contrary, it requires rather extensive length for the $3 \mathrm{D}$ reconstruction phase, using efficient computers for several hours. Otherwise, the technology used by the examined app takes a few minutes to capture and a few seconds for the object's 3D reconstruction, textured, and ready to be shared. For this reason, this technology can be considered low-cost par excellence, either because it uses a device mostly used for its core functionality, and because of the very low use of resources (in terms of skills required and in terms of computing times) to get critically analytical results.

\begin{tabular}{|l|c|c|}
\hline & 3D Creator app & $\begin{array}{l}\text { Canon /Xperia } \\
\text { XZ1- Photoscan }\end{array}$ \\
\hline Cost of equipment & + & + \\
\hline $\begin{array}{l}\text { Acquisition and } \\
\text { processing times }\end{array}$ & + & - \\
\hline $\begin{array}{l}\text { Training times } \\
\text { (easiness of uses, skills } \\
\text { required) }\end{array}$ & $+/-$ & - \\
\hline $\begin{array}{l}\text { Mesh quality } \\
\text { Accuracy (Hausdorff } \\
\text { distance evaluation) }\end{array}$ & - & + \\
\hline $\begin{array}{l}\text { Accessibility to the } \\
\text { acquired data }\end{array}$ & + & + \\
\hline
\end{tabular}

Table 1. Comparison between $3 D$ Creator app and Photoscan procedures.

Table 1 shows the main parameters considered for the surface model quality assessment, with a comparison of the used procedures. By analysing the report, we can state that whenever metric accuracy and mesh detail are not required (visualization purposes), the rapid method incorporated by the device used generally can be preferred. Regarding accessibility to the required data, it should be noted that the Xperia XZ1 smartphone has a real-time outline of the outcomes (available for major social platforms and as a stand-alone digital file).

One aspect introduced in the Sony application is the possibility of directly obtaining the three-dimensional print of the digital model, without further elaboration or control. In developing this first field experience, it will be interesting to evaluate how rapid reverse modelling and prototyping can be triggered from these 
kinds of tools such as ways, qualities and possible uses, in accordance with a growing metric quality that the future versions will probably allow.

In the future we will work to semantically enrich these models creating smart 3D repositories in order to query them with respect to geometrical instances, topological issues and historical features. This last aspect, (archives and Web 2.0 for data management closely connected with geometrical aspects), lead our digital model to a new dimension, the informative one (where spatial, temporal, historical and building parameters work together), that should always characterise speculative actions towards the constitution of the wealth of knowledge. We need to work on the efficiency of the process to reach effectiveness of the survey methodologies.

\section{ACKNOWLEDGMENTS}

This work has been carried out according to a memorandum of interest between University of Catania and the Department of Cultural Heritage of the City Hall of Catania. Authors would like to thank dott. Valentina Noto director of the Museo Civico Castello Ursino in Catania and the staff, in particular dott. Floriana Cappadona, as well as the students Raissa Garozzo, Damiano Aiello and Federico La Russa for helping during the acquisition process.

\section{REFERENCES}

Allegra, D., Gallo, G., Inzerillo, L, Lombardo, M., Milotta, F.L.M., Santagati, C., Stanco, F., 2017. Hand Held 3D Scanning for Cultural Heritage: Experimenting Low Cost Structure Sensor Scan. In Ippolito A. (ed.), Handbook of Research on Emerging Technologies for Architectural and Archaeological Heritage, IGI Global, pp 475-499.

Apollonio, F.I., Gaiani, M., Remondino, F., 2010. Una pipeline per l'acquisizione di dati 3D. In: Model. Digit. 3D Archeol. Caso Di Pompei. Edizioni della Normale, Pisa.

Benedetti, B., Gaiani, M., Remondino, F., 2010. Modelli digitali 3d in archeologia: il caso di Pompei, Strumenti, Edizioni della Normale, Pisa.

Bostanci, E., Kanwal, N., Clark, A.F., 2015. Augmented reality applications for cultural heritage using Kinect, Human-Centric Comput. Inf. Sci. 5.

Chiabrando, F., Lo Turco, M., Santagati, C., 2017. Digital invasions: from point clouds to Historical Building Object Modeling (H-BOM) of a UNESCO WHL site. In: The International Archives of the Photogrammetry, Remote Sensing and Spatial Information Sciences, vol. XLII-2 n. W3, pp. 171-178.

Clini, P., Frapiccini, N., Mengoni, M., Nespeca, R., Ruggeri, L., 2016. SFM technique and focus stacking for digital documentation of archaeological artifacts. In: Int. Arch. Photogramm. Remote Sens. Spat. Inf. Sci. - ISPRS Arch., pp. 229-236.

Evgenikou, V., Georgopoulos, A., 2015. Investigating 3D reconstruction methods for small artifacts. In: Int. Arch. Photogramm. Remote Sens. Spat. Inf. Sci., pp. 101-108.

Lachat, E., Landes, T., Grussenmeyer, P, 2017, Performance investigation of a handheld $3 \mathrm{~d}$ scanner to define good practices for small artefact $3 \mathrm{~d}$ modeling. In: The International Archives of the Photogrammetry, Remote Sensing and Spatial Information Sciences, vol. XLII-2/W5, pp. 427-434.
Kersten, T. P., Lindstaedt, M., 2012a. Automatic 3D object reconstruction from multiple images for architectural, cultural heritage and archaeological applications using open-source software and web services, Photogramm, Fernerkundung Geoinf, 6, pp. 727-740.

Kersten, T. P., Lindstaedt, M., 2012b. Image-based low-cost systems for automatic 3D recording and modelling of archaeological finds and objects. In: Lect. Notes Comput. Sci. (Including Subser. Lect. Notes Artif. Intell. Lect. Notes Bioinformatics), pp. 1-10.

Kurzydlo, W., Stach, B., Bober, A., Wodzinska, M., Dlugosz, M.M., 2014. Structured-light 3D scanner in use to assess the human body posture in physical therapy - a pilot study., Folia Med. Cracov. 54, pp. 21-35.

Li, W., Huhns, M.N., Tsai, W., and Wu, W. (eds), 2016. Crowdsourcing: Cloud-based Software Development, SpringerVerlag, Berlin.

Pagliari, D., Pinto, L., 2015. Calibration of Kinect for Xbox One and Comparison between the Two Generations of Microsoft Sensors, Sensors. 15 27569-27589.

Remondino, F., 2011. Heritage Recording and 3D Modeling with Photogrammetry and 3D Scanning. Remote Sensing, 3(6), pp. 1104-1138.

Richards-Rissetto, H., Remondino, F., Agugiaro, G., Von Schwerin, J., Robertsson, J., Girardi, G.. 2012. Kinect and 3D GIS in archaeology. In: Proc. 2012 18th Int. Conf. Virtual Syst. Multimedia, VSMM 2012 Virtual Syst. Inf. Soc., pp. 331-337.

Santagati C., Inzerillo L., Di Paola F., 2013. Image-based modeling techniques for architectural heritage $3 \mathrm{~d}$ digitalization: limits and potentialities, in The International Archives of the Photogrammetry, Remote Sensing and Spatial Information Sciences, Vol. XL-5/W2, 20. pp. 550-560.

Santagati, C., Inzerillo, L., 2016. Crowdsourcing Cultural Heritage: From 3D Modeling to the Engagement of Young Generations. EuroMed 2016: Digital Heritage. Progress in Cultural Heritage: Documentation, Preservation, and Protection, pp. 869-879.

Silberman, N., Fergus, R., 2011. Indoor scene segmentation using a structured light sensor. In: 2011 IEEE Int. Conf. Comput. Vis. Work.ICCV Work., pp. 601-608.

Smisek, J., Jancosek, M., Pajdla, T., 2011. 3D with Kinect. In: 2011 IEEE Int. Conf. Comput. Vis. Work.ICCV Work., IEEE, pp. 1154-1160.

Tong, J., Zhou, J., Liu, L., Pan, Z., Yan, H., 2012. Scanning 3D full human bodies using kinects, IEEE Trans. Vis. Comput. Graph. 18, pp. 643-650.

Vivacqua, R., Vassallo, R., Martins, F., 2017. A Low Cost Sensors Approach for Accurate Vehicle Localization and Autonomous Driving Application. In: Sensors. 17, pp. 1-33.

Ward, E., Folkesson, J., 2016. Vehicle localization with low cost radar sensors. In: IEEE Intell. Veh. Symp. Proc., pp. 864870. 